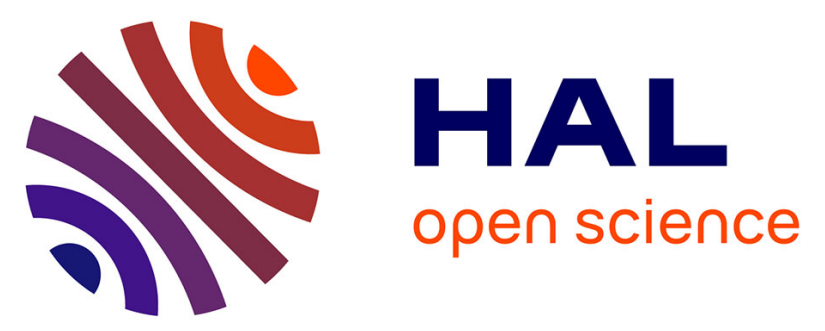

\title{
Lu5Ir4Si10 whiskers: Morphology, crystal structure, superconducting and charge density wave transition studies
}

Christine Opagiste, Maxime Leroux, Pierre Rodiere, G. Garbarino, Sébastien Pairis, Pierre Bordet, Pascal Lejay

\section{To cite this version:}

Christine Opagiste, Maxime Leroux, Pierre Rodiere, G. Garbarino, Sébastien Pairis, et al.. Lu5Ir4Si10 whiskers: Morphology, crystal structure, superconducting and charge density wave transition studies. Journal of Crystal Growth, 2010, 312, pp.3204-3208. 10.1016/J.JCRYSGRO.2010.07.037 . hal00564398

\section{HAL Id: hal-00564398 https://hal.science/hal-00564398}

Submitted on 9 Feb 2011

HAL is a multi-disciplinary open access archive for the deposit and dissemination of scientific research documents, whether they are published or not. The documents may come from teaching and research institutions in France or abroad, or from public or private research centers.
L'archive ouverte pluridisciplinaire HAL, est destinée au dépôt et à la diffusion de documents scientifiques de niveau recherche, publiés ou non, émanant des établissements d'enseignement et de recherche français ou étrangers, des laboratoires publics ou privés. 
Ref : J. Cryst. Growth 312, 3204 (2010)

\title{
$\mathrm{Lu}_{5} \mathrm{Ir}_{4} \mathrm{Si}_{10}$ whiskers: morphology, crystal structure, superconducting and charge density wave transition studies
}

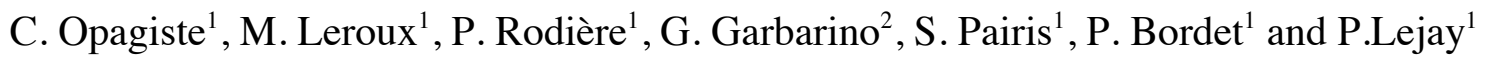 \\ ${ }^{1}$ Institut Néel, CNRS / UJF, PB 166, 38042 Grenoble Cedex 9, France \\ ${ }^{2}$ European Synchrotron Radiation Facility (ESRF), BP 220, 6 Rue Jules Horowitz, \\ 38043 Grenoble Cedex, France
}

\begin{abstract}
Highly perfect single crystal whiskers of $\mathrm{Lu}_{5} \mathrm{Ir}_{4} \mathrm{Si}_{10}$ were successfully grown out of the melt. Details of the surface and morphology of the whiskers are presented. X-ray diffraction data confirmed that the whisker structure has the same tetragonal $\mathrm{P} 4 / \mathrm{mbm}$ space group symmetry as bulk single crystals with lattice parameters a = 12.484(1) $\AA$ and $c=4.190(2) \AA$. By means of Field Emission Scanning Electron Microscopy, the morphology of the whiskers has been studied. Using X-ray Laue technique we found that whiskers grow along the c axis direction and all side faces are oriented along the [110] direction. The mosaicity has been measured and is found to be almost perfect: below $0.15^{\circ}$ along the $\mathrm{c}$ axis. According to our transport measurements performed along the c axis, the whiskers present a sharp superconducting transition at $\mathrm{Tc}=4.1 \mathrm{~K}$ and show a Charge Density Wave (CDW) transition at $77 \mathrm{~K}$. From the hysteresis of the temperature dependance of the electrical resistivity study, the CDW transition is found to be of first order.
\end{abstract}

Keywords: A1. Crystal structure; A1. X-ray diffraction; A2. Growth from melt; A2. Single crystal growth; B1.Rare earth compounds; B2. superconducting materials

PACS classification codes: $61.50 . \mathrm{Ah} ;$ 61.05.C- ; 81.10.Fq ; 68.70.+w ; 72.15.Eb ; 74.25.F-

\section{Introduction}

The compounds of the intermetallic series $R_{5} \operatorname{Ir}_{4} \mathrm{Si}_{10}$ with $R=$ rare-earth exhibit a behaviour where either superconductivity and Charge Density Wave (CDW) ordering or magnetic ordering and CDW ordering coexist in the same material depending on the magnetic or non magnetic nature of the rare earth element $R[1-6]$. Among them, the $\mathrm{Lu}_{5} \mathrm{Ir}_{4} \mathrm{Si}_{10}$ compound becomes superconducting at $4 \mathrm{~K}$ and shows a strongly coupled CDW transition around $80 \mathrm{~K}[1-4]$. Like the other compounds of those series, $\mathrm{Lu}_{5} \mathrm{Ir}_{4} \mathrm{Si}_{10}$ crystallizes in the tetragonal $\mathrm{Sc}_{5} \mathrm{Co}_{4} \mathrm{Si}_{10}$ type structure with space group $\mathrm{P} 4 / \mathrm{mbm}$ [7]. Single crystals of $\mathrm{Lu}_{5} \mathrm{Ir}_{4} \mathrm{Si}_{10}$ are generally grown in a three-arc furnace using a modified Czochralski technique $[3,8]$ or by 
Ref : J. Cryst. Growth 312, 3204 (2010)

the floating zone technique using an image furnace in Ar atmosphere [9]. In our process to obtain bulk single crystal grown with the modified Czochralski technique, we have observed a spontaneous growth of whiskers out of the under cooled melt. In this paper we describe the morphology, the crystallographic structure refinement and the transport property measurements performed on $\mathrm{Lu}_{5} \mathrm{Ir}_{4} \mathrm{Si}_{10}$ whiskers.

\section{Experimental}

A polycrystalline sample of $\mathrm{Lu}_{5} \mathrm{Ir}_{4} \mathrm{Si}_{10}$ was prepared in an induction furnace, in a cold copper crucible and in a high purity argon atmosphere, with a stoichiometric amount of Lu (99.96\%, Ames Lab.), Ir (99.9\%, Alfa Aesar) and Si (99.9999\%, Alfa Aesar). The sample was melted several times to improve its homogeneity. Mass losses during these first steps were less than $0.1 \%$. The polycrystalline sample was then placed in a three-arc furnace in a water cooled copper crucible and in a purified argon atmosphere. The cooling speed seems to be an important parameter for the growth of whiskers. Whiskers appear only when the melt is rapidly cooled in a fraction of a second, but not when the liquid phase is slowly cooled. Under convenient conditions, lots of whiskers can be seen emerging from the polycrystalline solid mass (fig. 1). A typical brittle needle-like single crystal is shown in the inset of figure 1.

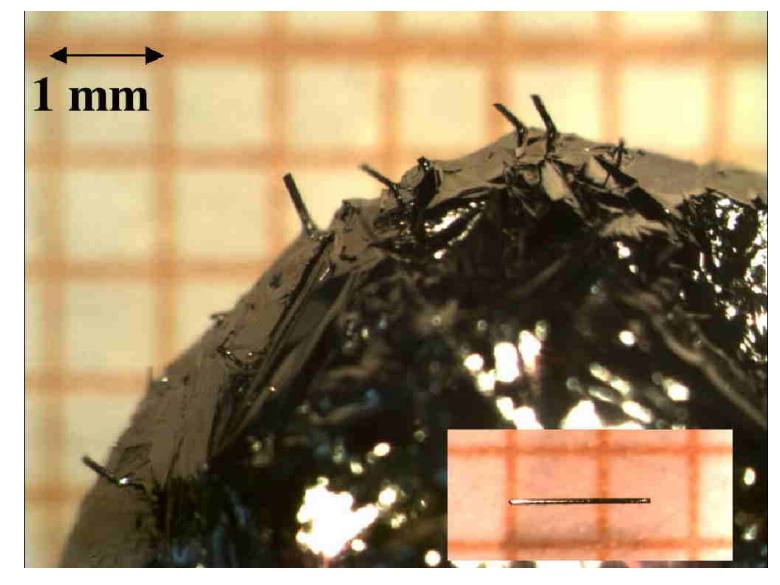

Figure 1:

whiskers can be observed emerging from the polycrystalline sample when this one is thermally quenched. In inset: a typical brittle needle-like whisker of $1.5 \mathrm{~mm}$ long.

\section{Results and discussion}

\subsection{Whiskers surface morphology, orientation and mosaicity}

From Field Emission Scanning Electron Microscopy observations (FESEM - Zeiss Ultra, all images presented in this paper are secondary electron images) we find that whiskers exhibit a very regular shape of a plate like crystal with typical dimensions: 15 x 60 x $1000 \mu \mathrm{m}^{3}$ (fig. 2). 
Ref : J. Cryst. Growth 312, 3204 (2010)

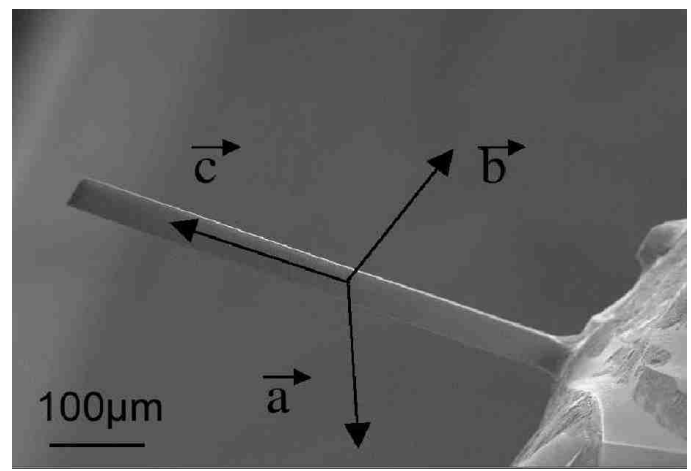

Figure 2: SEM image of a whisker. Orientation of the crystal axis is given. The face is oriented along the [110] direction.

We have never observed whiskers with a scroll shape. Observations of the surface reveal typical growth figures resulting of the formation of dendrites (fig. 3). This is not surprising since we have cooled the sample in a fraction of a second.
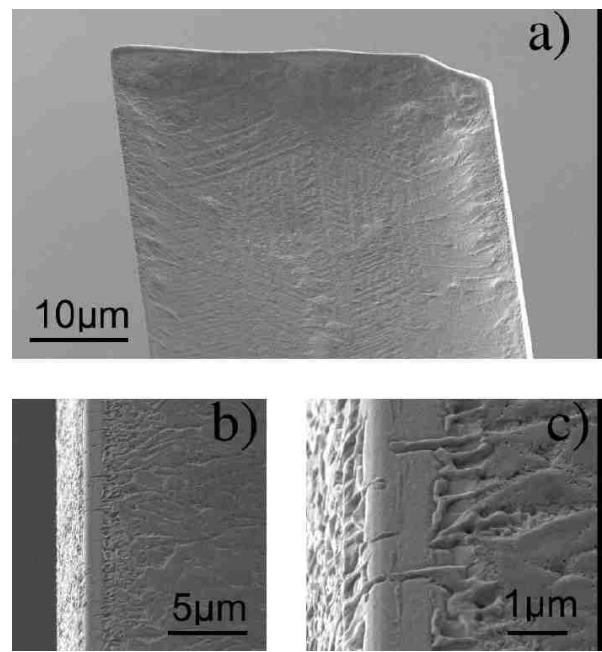

figure 3: (a) Detail of the extremity of a whisker showing a surface with signs of chevron-

shaped growth and sharp edges (b and c).

As seen on figure 4-a a growing dendrite is observed on the surface and above a cavity. The dendrite has one long branch lying on the surface with side branches in the same plane. We can distinguish the second and third orders of the dendrite branches (fig. 4-b). Typical whisker growth begins with a square pyramid (fig. 5) and has only been observed on the periphery of the polycrystalline thermally quenched sample. On the top surface of the polycrystalline sample we have never observed the formation of whiskers but only the formation of a multitude of square pyramidal concretions (fig 6). The extent to which the whiskers are not pointing out at the top depends on the mass transport and gravity and also on the temperature gradients. It is difficult to explain why the macroscopic structure of the whiskers looks like a plate like structure with a perfect rectangular cross section with sharp edges (fig. 3-b and 3-c). 
Ref : J. Cryst. Growth 312, 3204 (2010)
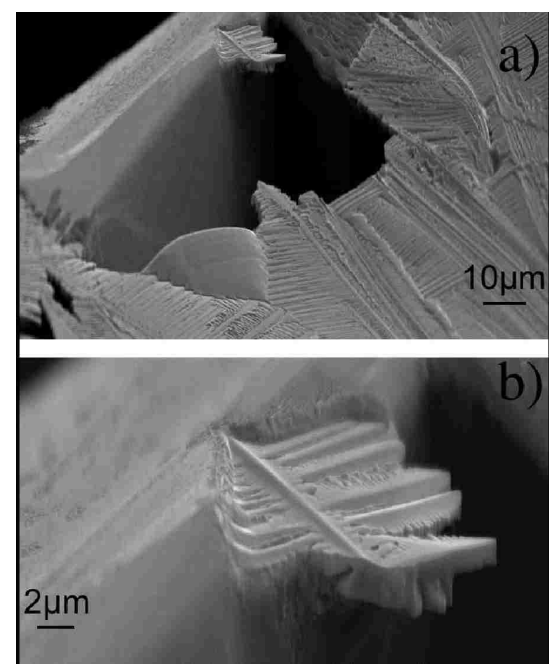

figure 4: (a) Dendrites observed on the surface of the polycrystalline sample. (b) Zoom

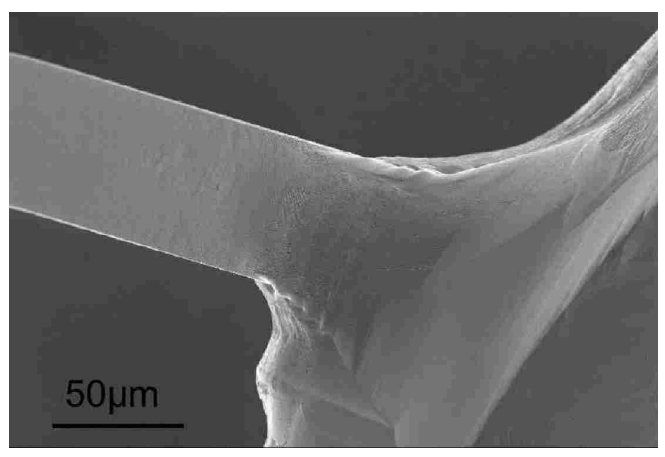

figure 5: A typical root of a whisker consists of a square pyramid.

The growth mechanism of whiskers is still controversial in the literature. It is generally accepted that the growth of whiskers is due to internal mechanical stress in the alloy during cooling. Even more, several authors claim that a screw dislocation is at the origin of their growth [10 - 12].

We have also performed Electron Back Scattered Diffraction (EBSD) analysis on different surfaces. Deduced from the pseudo Kikuchi resolved figures, the principal axis of the dendrites grows along the $\mathrm{c}$ axis direction.

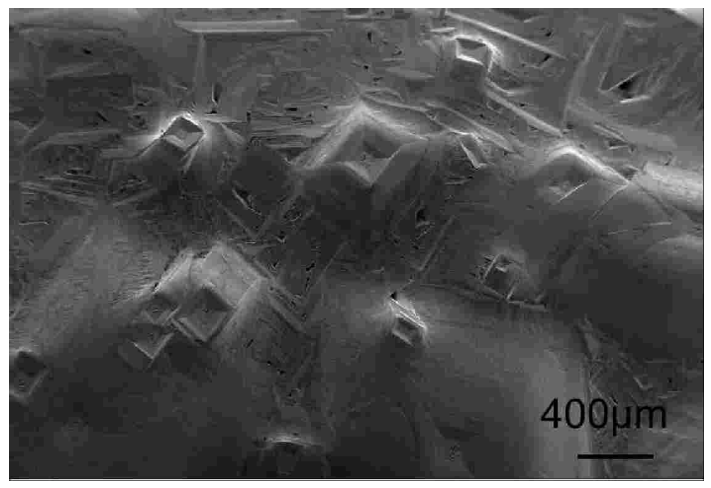

figure 6: SEM image of the top surface of the polycrystalline quenched sample. Many pyramidal concretions are observed. They do not give rise to the growth of whiskers. 
Ref : J. Cryst. Growth 312, 3204 (2010)

The surface formed by the dendrites growth is oriented along the [110] direction. It has been impossible to get such information on whiskers, due to the imperfect state of their surface and to the low thickness probed by EBSD technique. In order to get the whiskers orientation, we have used X-ray diffraction measurements. We have found that the whiskers grow along the $\mathrm{c}$ axis direction and that each surface is oriented along the [110] direction (see fig. 2).

In the inset of figure 7 a zoom of a diffraction image of the reflections (110) and (001) is shown, as well as the angle dependence of its intensities in semi-log scale. The measurements have been developed in the ID27 beam line of the ESRF in a transmition configuration using a MARCCD detector with a focused $\mathrm{X}$ ray beam of $3 * 3 \mu m^{2}$ at $33 \mathrm{keV}(\lambda=0.3738 \AA$ ). It can be observed the excellent crystalline quality of the whisker, both on the CCD image and on the angle dependence of the Bragg reflections. By a Gaussian fit of the intensity curves (red line on figure 7 (a) and (b)) we obtained that the mosaicity of the (110) and (001) reflections is better than $0.15^{\circ}$.

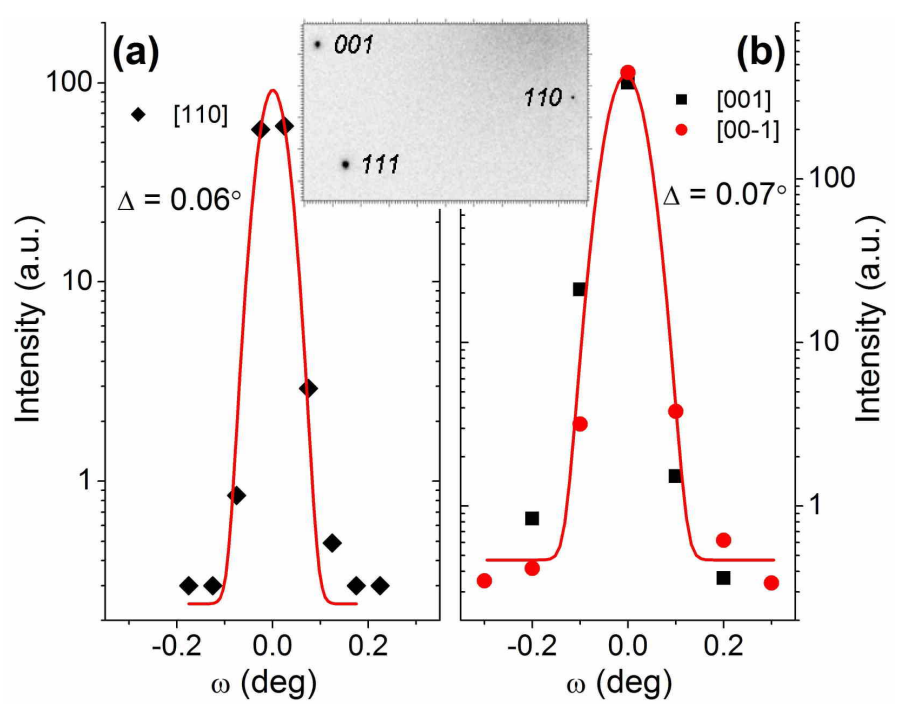

figure 7: Angle dependence of the (110) and $(00 \pm 1)$ reflections, panels $a$ and $b$, respectively (intensity is given in log scale). The half width half maximum of a Gaussian fit is represented by

$\Delta$ in both cases. It can be observed that the mosaicity of these two reflections is better than $0.15^{\circ}$. In the inset, a diffraction CCD image of the whisker is shown.

\subsection{X-ray diffraction refinement}

A whisker of dimensions $65 \times 30 \times 600 \mu \mathrm{m}^{3}$ was selected for $\mathrm{x}$-ray single crystal diffraction analysis, performed on a KappaApex II Bruker diffractometer equipped with a silver anode $(\lambda=0.5608 \AA)$ Incoatec $\mathrm{I} \mu \mathrm{S}$ microsource $\mathrm{x}$-ray generator. The data collection consists in 
Ref : J. Cryst. Growth 312, 3204 (2010)

a series of omega-scans covering $500 \mathrm{deg}$. at a speed of $15 \mathrm{~s} / \mathrm{deg}$. with a crystal to detector distance of $30 \mathrm{~mm}$. A primitive tetragonal unit cell was found with $\mathrm{a}=12.484(1) \AA$ and $\mathrm{c}=$ 4.190(2) A. The reflections were integrated using the EvalCCD suite [13] and anisotropic effects were corrected using the Sadabs program [14] leading to a Rint of $8.47 \%$ for all 1650 reflections averaged from 21612 measured ones. This rather high value of Rint is probably due to the high absorption $\left(\mu=425.9 \mathrm{~cm}^{-1}\right)$ and strongly anisotropic shape of the sample. Analysis of the systematic extinction conditions leads to the $\mathrm{P} 4 / \mathrm{mbm}$ space group. The structure was solved by direct methods using the Sir97 program [15]. The refinement was carried out using the Jana2000 program [16]. All atoms were refined with anisotropic atomic displacement parameters and an isotropic extinction correction, using a $1 / \operatorname{sig}(\mathrm{Fo})^{2}$ weighting scheme, where the $\operatorname{sig}(\mathrm{Fo})$ are the estimated standard deviation on observed structure factor moduli. The final agreement factors were Rwobs $=3.18 \%$ and Rwall $=3.39 \%$, gofobs $=1.86$ and gofall=1.86 (reflections are observed for $\mathrm{I}>3 . \operatorname{sig}(\mathrm{I})$ ). The positional and harmonic displacement parameters obtained are displayed in table 1 and 2.

These results are very close to those reported by Becker et al. [3] and obtained for a bulk single crystal.

Table 1: Atomic coordinates and isotropic displacement parameters for $\mathrm{Lu}_{5} \mathrm{Ir}_{4} \mathrm{Si}_{10}$.

\begin{tabular}{|l|l|c|c|c|c|}
\hline Atom & Wyckoff pos. & $\mathrm{X}$ & $\mathrm{Y}$ & $\mathrm{Z}$ & Uiso $\left(\AA^{2}\right)$ \\
\hline Ir & $8 \mathrm{i}$ & $0.018812(18)$ & $0.245589(17)$ & 0 & $0.00530(5)$ \\
\hline Lu1 & $2 \mathrm{a}$ & 0 & 0 & 0 & $0.00691(8)$ \\
\hline Lu2 & $4 \mathrm{~h}$ & $0.17411(2)$ & $0.32589(2)$ & 0.5 & $0.00760(6)$ \\
\hline Lu3 & $4 \mathrm{~h}$ & $-0.11531(2)$ & $0.38469(2)$ & 0.5 & $0.00815(6)$ \\
\hline Si1 & $4 \mathrm{~g}$ & $0.06628(15)$ & $0.43372(15)$ & 0 & $0.0064(4)$ \\
\hline Si2 & $8 \mathrm{i}$ & $0.19999(16)$ & $0.16501(16)$ & 0 & $0.0095(4)$ \\
\hline Si3 & $8 \mathrm{j}$ & $0.0035(2)$ & $0.15636(17)$ & 0.5 & $0.0157(5)$ \\
\hline
\end{tabular}

Table 2: Harmonic displacement parameters for $\mathrm{Lu}_{5} \mathrm{Ir}_{4} \mathrm{Si}_{10}$.

\begin{tabular}{|l|l|l|l|l|}
\hline Atom & $\mathrm{U} 11\left(\AA^{2}\right)$ & $\mathrm{U} 22\left(\AA^{2}\right)$ & $\mathrm{U} 33\left(\AA^{2}\right)$ & $\mathrm{U} 12\left(\AA^{2}\right)$ \\
\hline Ir & $0.00492(8)$ & $0.00477(8)$ & $0.00620(10)$ & $-0.00036(5)$ \\
\hline Lu1 & $0.00597(11)$ & $0.00597(11)$ & $0.00879(19)$ & 0 \\
\hline Lu2 & $0.00812(9)$ & $0.00812(9)$ & $0.00656(14)$ & $0.00196(10)$ \\
\hline Lu3 & $0.00802(9)$ & $0.00802(9)$ & $0.00841(14)$ & $0.00024(10)$ \\
\hline Si1 & $0.0057(5)$ & $0.0057(5)$ & $0.0079(9)$ & $0.0014(7)$ \\
\hline Si2 & $0.0076(7)$ & $0.0052(6)$ & $0.0158(8)$ & $0.0016(5)$ \\
\hline Si3 & $0.0316(12)$ & $0.0088(8)$ & $0.0068(7)$ & $-0.0048(8)$ \\
\hline
\end{tabular}

\subsection{Electrical resistivity measurements}

We carried out measurements of electrical resistivity from $300 \mathrm{~K}$ down to $2 \mathrm{~K}$ on a whisker. We used a traditional four-wire technique with an automatic resistance measurement apparatus TRMC2 [17]. The typical contact resistances were several Ohms. Around 77K, the calibration of the thermometer was checked with the boiling temperature of nitrogen at ambient 
Ref : J. Cryst. Growth 312, 3204 (2010)

pressure. Moreover, to check the absence of any self-heating, different current densities ranging from $0.02 \mathrm{~A} / \mathrm{cm}^{2}$ to $20 \mathrm{~A} / \mathrm{cm}^{2}$ were used.

The resistivity of the whisker is shown figure 8 . This is quantitatively the same temperature dependence as in c-axis bulk samples reported in the literature [3].

Below $76.6 \mathrm{~K}$, a clear jump on the resistivity of $30 \%$ is due to the formation of the Charge Density Wave. A highly reproducible temperature hysteresis of $1 \mathrm{~K}$ wide has been measured. The same width has been measured with different speeds ranging from $50 \mathrm{mK} / \mathrm{min}$ to $500 \mathrm{mK} / \mathrm{min}$. The hysteresis appears over the whole transition jump contrary to previous report on bulk sample [18]. This hysteresis is a clear evidence of the $1^{\text {st }}$ order nature of the transition previously reported [3]. At lower temperature a full superconducting transition is measured. The critical temperature is $4.1 \mathrm{~K}(50 \%$ of the residual resistivity) and $35 \mathrm{mK}$ wide $(10 \%-90 \%$ criterion). No significant changes were observed with current densities from $20 \mathrm{~A} / \mathrm{cm}^{2}$ down to $0.02 \mathrm{~A} / \mathrm{cm}^{2}$.

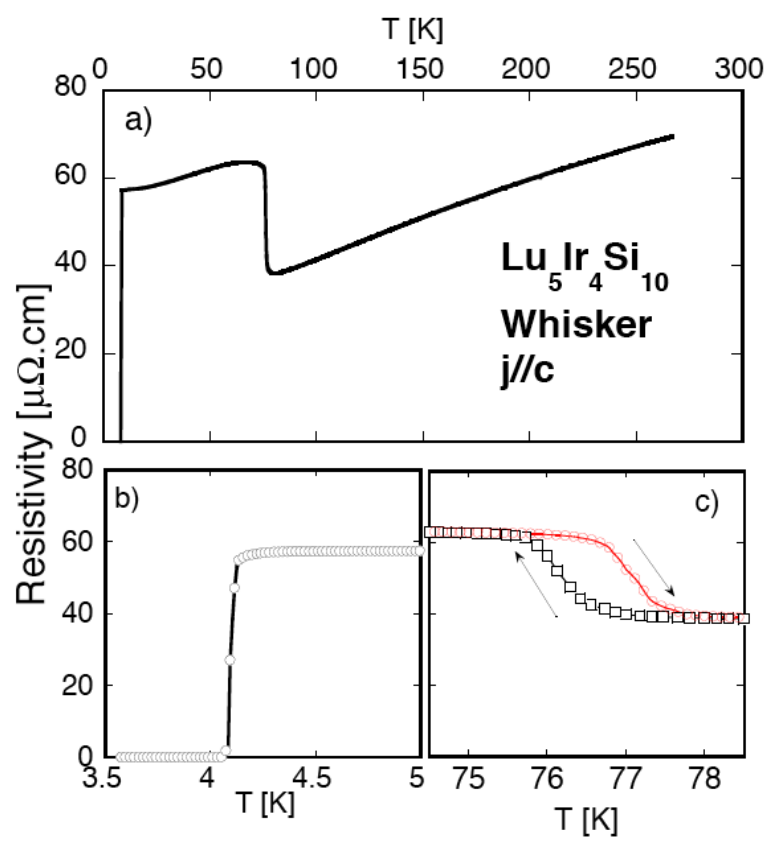

Figure 8: Temperature dependence of the electrical resistivity of a whisker of $\mathrm{Lu}_{5} \mathrm{Ir}_{4} \mathrm{Si}_{10}$ along caxis with a current density of $20 \mathrm{~A} / \mathrm{cm}^{2}$. a) from room temperature to $2 \mathrm{~K}$

b) zoom on the superconducting transition at $4.1 \mathrm{~K}$

c) zoom on the temperature hysteresis at the Charge Density Wave transition around 76.6K.

\section{Conclusion}

High quality single crystal whiskers of $\mathrm{Lu}_{5} \mathrm{Ir}_{4} \mathrm{Si}_{10}$ were successfully grown. They exhibit a very regular shape of a plate like crystal with typical dimensions 15 x 60 x $1000 \mu \mathrm{m}^{3}$. X-ray diffraction data confirmed that whiskers grow along the c direction and the faces are oriented 
Ref : J. Cryst. Growth 312, 3204 (2010)

along the [110] direction. The mosaicity has been measured and is found to be almost perfect: below $0.15^{\circ}$.

The structure of the whisker was resolved and we confirmed that whiskers crystallise in the same tetragonal $\mathrm{P} 4 / \mathrm{mbm}$ space group as bulk single crystal obtained by different techniques. Lattice parameters are $\mathrm{a}=12.484(1) \AA$ and $\mathrm{c}=4.190(2) \AA$.

Electrical resistivity versus temperature measurements performed with current apply along the whisker axis show the same typical dependence as c-axis bulk sample. Whiskers have a sharp superconducting transition at $4.1 \mathrm{~K}$ and a $\mathrm{CDW}$ transition at $77 \mathrm{~K}$. A temperature hysteresis study has confirmed the first order nature of the CDW transition.

Acknowledgments: We greatly acknowledge the technical assistance of Joël Balay and Abdellali Hadj-Azzem from the Crystal Growth Service at the Néel Institut.

\section{Références :}

[1] H. D. Yang, R. N. Shelton, and H. F. Braun, Phys. Rev. B 33 (1986 ) 5062.

[2] R.N. Shelton, et al. Phys. Rev. B 34 (1986) 4590.

[3] B. Becker, et al. Phys. Rev. B 59 (1999) 7266.

[4] Y. Singh, R. Nirmala, S. Ramakrishnan, and S. K. Malik, Phys Rev. B 72 (2005) 045106.

[5] H. D. Yang, P. Klavins, and R. N. Shelton, Phys. Rev. B 43 (1991) 7688.

[6] K. Ghosh, S. Ramakrishnan, and G. Chandra, Phys. Rev. B 48 (1993)4152.

[7] H. F. Braun, K. Yvon and R. H. Braun, Acta Crystallogr. B 36 (1980) 2397.

[8] J. B. Betts, et al. Phys. Rev. B 66 (2002) 060106(R).

[9] T. Tamegai and G.J. Li, Int. J. Modern Phys. B Vol. 21 nº18-19 (2007) 3334.

[10] J.D. Eshelby, J. Appl. Phys. 24 (1953) 176.

[11] A. Menovsky, J.J.M. Franse and A.C. Moleman, J. Crystal Growth 67 (1984) 31.

[12] B. Ellman, A. Zaluska, L. Taillefer, Physica B 205 (1995) 346.

[13] A.J.M. Duisenberg, et al., J. Appl. Cryst. 36 (2003) p220-229.

[14] R.H. Blessing, Acta Cryst. A51 (1995) 33-38

[15] Altomare, A., Burla, et al.; J. of Appl. Cryst. 32 (1999) 115.

[16] Petricek V.,et al Jana2000. Struct. Det. Software Programs. Inst. of Physics, Praha, Czech Republic.

[17] TRMC2 resistance bridge, http://neel.cnrs.fr/spip.php?article862

[18] S. Ramakrishnan, J.A. Mydosh, JMMM 310 (2007) 207-213. 